\title{
Diacronie
}

Studi di Storia Contemporanea

$N^{\circ} 19,3 \mid 2014$

Miscellaneo

\section{«The Family of Man»: réflexions autour des usages et de la patrimonialisation d'une exposition photographique controversée}

\section{Claude Dupuis}

\section{(2) OpenEdition \\ Journals}

Édition électronique

URL : http://journals.openedition.org/diacronie/1582

DOI : 10.4000/diacronie. 1582

ISSN : 2038-0925

Éditeur

Association culturelle Diacronie

Référence électronique

Claude Dupuis, « «The Family of Man»: réflexions autour des usages et de la patrimonialisation d'une exposition photographique controversée », Diacronie [En ligne], N¹9, 3 | 2014, document 3, mis en ligne le 01 septembre 2014, consulté le 01 mai 2019. URL : http://journals.openedition.org/ diacronie/1582 ; DOI : 10.4000/diacronie.1582 


\section{Diacronie}

N. 19 | $3 \mid 2014$

\section{3/}

\section{«The Family of Man»: réflexions autour des usages et de la patrimonialisation d'une exposition photographique controversée}

Claude DUPUIS *

Entre sa création en 1955 et sa disparition temporaire en 1966, l'exposition photographique conçue par Edward Steichen à partir d'images collectées à travers le monde n'a cessé d'attirer un public nombreux. Restaurée dans les années '90 et présentée de manière permanente au Grand-Duché du Luxembourg depuis juin 1994, l'exposition véhicule depuis son origine un message "humaniste" différemment apprécié des critiques. La présente contribution revient dans un premier temps sur ces différentes polémiques, principalement animées par le rejet d'un américanisme à la fois trop libéral et trop religieux et moralisateur. Parallèlement elle tente de dissocier les usages qui ont pu être par les propriétaires successifs de l'exposition, de sa circulation pendant la Guerre Froide à son installation au Château de Clervaux. A partir de l'hypothèse d'une utilisation précédemment "nationaliste" de la collection photographique, c'est enfin une réflexion sur la genèse et les enjeux de l'inscription de "The Family of Man » au "Registre de la Mémoire du Monde" de l'UNESCO en 2003 qui est menée.

\section{Introduction}




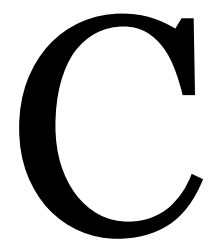

réée en 1955, régulièrement qualifiée "d'humaniste et universelle", puis déclinée en plusieurs versions qui parcourront le monde, l'exposition photographique «The Family of Man» a été et reste encore souvent considérée comme un outil de propagande au service des Etats-Unis d'Amérique dans ces périodes cruciales pour sa politique internationale que représentent l'Après-guerre et la Guerre Froide. Désormais l'unique version itinérante encore existante est conservée au Grand-Duché du Luxembourg et a été inscrite en 2003 au "Registre de la Mémoire du Monde" de l'UNESCO. Dans un premier temps notre contribution reviendra brièvement sur l'histoire de l'exposition. Ensuite elle s'intéressera aux diverses réactions suscitées par l'exposition avant d'envisager les usages qui lui ont été attribués. Dans un dernier temps, c'est sur les divers enjeux de la patrimonialisation de cette exposition hors-du-commun que se portera toute notre attention.

\section{L'exposition photographique « The Family of Man »}

\subsection{Bref rappel historique}

C'est assisté par Wayne Miller qu'Edward J. Steichen, peintre et photographe d'origine luxembourgeoise, crée en janvier 1955 l'exposition photographique « The Family of Man » au MoMA, le musée d'art moderne de New York au sein duquel il occupe le poste de directeur du département de la photographie depuis 1947. Après plusieurs années de collecte, Steichen propose là une exposition ne comprenant pas moins de 503 clichés issus de 273 photographes professionnels ou non, racontant ainsi en images et en 37 chapitres l'expérience de la vie humaine de la naissance à la mort ${ }^{1}$. Entre 1955 et 1962 l'exposition est déclinée en plusieurs versions itinérantes. C'est grâce notamment au soutien de l'USIA (United States Information Agency) que «The Family of Man» peut ainsi circuler à travers le monde ${ }^{2}$. Tous les continents accueillent l'exposition même si l'Afrique fait en quelque sorte figure d'exception : seuls les futurs Zimbabwe et Kenya ainsi que l'Afrique du Sud, tous alors sous emprise britannique, reçoivent « The Family of Man ». Fin 1964 le gouvernement des Etats-Unis propose au

\footnotetext{
${ }^{1}$ Cfr. Mémoire du Monde: Family of Man, URL :

$<\quad$ http://www.unesco.org/new/fr/communication-and-information/flagship-projectactivities/memory-of-the-world/register/full-list-of-registered-heritage/registered-heritagepage-3/family-of-man/ > [consulté le 11 février 2014].

2 WULF, Andrew, Moscow '59 The "Sokolniki Summit" Revisited, Los Angeles, Figueroa Press, 2010, p. 10.
} 
Grand-Duché de lui offrir cette dernière version itinérante de l'exposition. Celle-ci est alors présentée au public luxembourgeois en 1965 puis en 1966 lors de la venue d'Edward Steichen qui plaide en faveur du Château de Clervaux pour abriter définitivement cette collection de photographies.

\subsection{Les versions}

La version aujourd'hui inscrite au "Registre de la Mémoire du Monde" est la version européenne3 ${ }^{3}$ C'est aussi la plus complète. Il reste possible que des parties d'autres versions soient stockées dans des endroits aujourd'hui oubliés. Les variations entre la version new-yorkaise originale et celle conservée au Château de Clervaux, au GrandDuché du Luxembourg 4 sont repérables à l'examen de l'unique catalogue jamais réalisé pour cette exposition 5 . Dans une interview réalisée en 1993 Yoshio Watanabe est revenu sur l'organisation de l'exposition à Tokyo en 1956 et l'influence de Steichen dans ces variations. L'ancien membre du comité exécutif de l'exposition dans la capitale japonaise a expliqué comment le curateur américain avait fait ajouter une soixantaine d'œuvres japonaises et son homologue japonais en avaient retirées d'autres par crainte "de remous " ${ }^{6}$. Goro Kuramochi a quant à lui révélé que Steichen aurait approuvé cette éviction?.

En 1959 c'est à Moscou qu'est présentée l'exposition dans le cadre plus vaste de l'American National Exhibition. Connue grâce au Kitchen Debate ${ }^{8}$ cette exposition ou

3 Cfr. Dossier de proposition d'inscription rédigé par JeanBack, p. 2. URL: < http://www.unesco.org/new/fileadmin/MULTIMEDIA/HQ/CI/CI/pdf/mow/nomination_for ms/Family\%20of\%20Man\%20\%20Nomination\%20FormFR.pdf > [consulté le 11 février 2014]. 4 D'une part la version conservée n'est pas complète puisque 18 images ont été perdues avant leur arrivée au Grand-Duché, d'autre part certaines photographies de la version originale ont été remplacées dans la perspective d'une présentation en dehors des Etats-Unis. D'après Gabriele et Helmut Nothelfer, une seule image du ghetto de Varsovie a été exposée à Berlin en 1955. À Clervaux aujourd'hui il y en a deux. La photographie de cet enfant juif dans le ghetto de Varsovie en Mai 1943, issue du rapport de Jürgen Stroop, commandant des troupes allemandes lors de sa destruction n'apparait pas dans le catalogue. Elle a donc été ajoutée dans l'une des versions. Cfr. DUPUIS, Claude, «The Family of Man et son flow d'images: un labyrinthe socio photographique?», in Revue de l'Institut de Sociologie 2010-2011, 1-4/2012, p. 278. NOTHELFER, Gabriele, NOTHELFER, Helmut, "1955 "Nous tous - The Family of Man à Berlin"», in The Family of Man. Témoignages et documents, Luxembourg, Editions Artevents, 1994, p. 143.

5 The Family of Man, created by Edward STEICHEN, prologue by Carl SANDBURG, New York, The Museum of Modern Art, 1955.

${ }^{6}$ «Un des problèmes qui se sont posés au moment de la préparation fut les photos de la bombe atomique, dues à Yamahata. L'empereur devait visiter l'exposition, et la question se posait de savoir si on montrerait ou non ces photos». KURAMOCHI, Goro, WATANABE, Yoshio, «Tokyo 1956», in The Family of Man. Témoignages et documents, cit., pp. 150-151.

7 Ibidem, pp. 151-152.

8 Cfr. New-York Times du 25 juillet 1969. URL : 
plutôt cette foire commerciale présentait à la fois une large gamme de la production industrielle américaine mais aussi des artistes ou expositions comme «The Family of Man». Le but était de présenter "les avantages" de la vie domestique américaine 9 . Entre sa première présentation à Berlin en 1955, son passage à Paris et Tokyo en 1956, à Bruxelles en 1958 et celle de Moscou où contrairement aux autres étapes de son périple mondial l'exposition n'était pas un objet entièrement autonome en soi, il faut en envisager différents usages, lesquels furent souvent sujets de vives critiques ou de controverses.

\subsection{Usages, critiques et controverses}

Dans l'espace francophone, c'est Roland Barthes qui, dans son ouvrage Mythologies paru en $1957^{10}$, a émis avec « La grande famille des hommes » la critique à la fois la plus reprise et encore très commentée aujourd'hui. Le philosophe reproche à l'équipe créatrice, en particulier à Edward Steichen, de promouvoir un universalisme de la nature humaine en s'appuyant sur un humanisme issu d'un dogme religieux. Il déplore de surcroit l'absence de vision historique, ce qui équivaut, même si cela n'est pas ouvertement énoncé, à une négation de toute évolution. C'est aussi ce qui ressort de l'analyse de l'exposition à Berlin par Gabriele et Helmut Nothelfer ${ }^{11}$. Il faut signaler que les expressions retenues pour présenter « The Family of Man » à travers le monde furent souvent tirées du vocabulaire religieux. Dans la préface du catalogue berlinois, Steichen est assimilé au «Schöpfer » [Créateur] et le vocable « Glaube » [foi] apparait dans chaque phrase ${ }^{12}$. Déjà Barthes avait souligné ce recours à Dieu dans la

< http://www.nytimes.com/learning/general/onthisday/big/o724.html\#article > [consulté le 28 février 2014]; VOSSOUGHIAN, Nader, The Conference in the Kitchen: Domesticity, Cold War Politics, and the American National Exhibition in Moscow (1959), URL:

<http://www.academia.edu/3127492/The_Conference_in_the_Kitchen_Domesticity_Cold_W ar_Politics_and_the_American_National_Exhibition_in_Moscow_1959_ > [consulté le 28 février 2014].

9 «It is billed by the USIA as containing "cultural, scientific and technological exhibits designed to further Soviets understanding life in America" »[Il est considéré par l'USIA comme contenant " des objets culturels, scientifiques et technologiques conçus pour faire davantage comprendre aux soviétiques de la vie en Amérique »]. The American National Exhibition, Moscow, 1959. Hearings before the committee on UN-American activities House of representatives, Eighty-six congress, first session, July 1, 1959. Printed for the use of the Committee on Un-American Activities, Washington, United states government printing office, 1959, p. 895.

${ }^{10}$ BARTHES, Roland, La grande famille des hommes, in ID., Mythologies, Paris, Le Seuil, 1970, p. 806.

${ }^{11}$ NOTHELFER, Gabriele, NOTHELFER,Helmut, op. cit., pp. 141-145.

12 Document: préface du catalogue de l'exposition de Berlin, in The Family of Man. Témoignages et documents, cit., p. 140. 
présentation française de l'exposition ${ }^{13}$. C'est dans le même registre qu'il faut classer la présentation de l'exposition dans le numéro de février 1956 du Courrier de l'Unesco ${ }^{14}$. Après une brève introduction sous-titrée «vaste symphonie photographique», c'est une traduction du texte introductif au catalogue signé de Carl Sandburg, poète et beau-frère de Steichen, qui accompagne les différentes photographies extraite de La grande famille des Hommes. Un texte signé Cyril Bibby sur l'enseignement des races aux enfants vient s'ajouter au tout. Les nombreuses références religieuses de Sandburg, les mots de l'introduction dans le «Courrier de l'Unesco» empruntés à André Maurois «s'aimer les uns les autres...»15 ou ceux d'André Chamson rapportés par Barthes font que cette entreprise a été et reste souvent considérée comme un message dévot. En 2005 Rosch Krieps s'efforçait de combattre cette critique persistante dans son guide de visite de l'exposition à l'intention des publics scolaires luxembourgeois. S'appuyant sur les derniers mots de Steichen en introduction au catalogue, il réfute le contenu religieux du message véhiculé par l'exposition ${ }^{16}$. L'écrivain Michel Tournier a, sans écarter le religieux et en comparant l'acte photographique à une création divine, résumé les deux positionnements idéologiques qui continuent de s'affronter: «On peut se laisser gagner par le souffle spirituel qui parcourt ces pages [l'exposition] mais on peut également s'irriter des prétentions moralisantes de ce prêchi-prêcha» ${ }^{17}$.

Une autre critique tout aussi persistante est que l'exposition servit d'outil de propagande aux États-Unis d'Amérique. C'est ce qui probablement explique l'insistance des différents auteurs plus sur la présentation moscovite de l'exposition que sur les moyens déployés par la USIA pour faire circuler l'exposition à travers le monde ${ }^{18}$. Eric Sandeen considère même que l'étape dans la capitale soviétique fut la plus importante

13 BARTHES, Roland, op. cit., p. 162.

${ }^{14}$ Le courrier de l'Unesco, IX, février 1956, pp. 18-33. URL :

< http://unesdoc.unesco.org/images/ooo6/o00682/068278fo.pdf > [consulté le 30 mars 2014].

${ }^{15}$ Le courrier de l'Unesco, cit., p. 19.

16 « Manche Leute wollen THE FAMILY OF MAN als eine Art moderne Bilderbibel, als religiöses Werk einstuffen, was aber angesichts der allein dem „Vertrauen auf die Menschheit“ gewidmeten Schlussfolgerung von Steichen selbst nicht zutrifft » [Quelques personnes veulent considérer The Family of Man comme une sorte de bible imagée moderne, une œuvre religieuse, ce qui ne correspond pas, au regard de la seule dédicace "Confiance en l'Humanité " à la conclusion de Steichen]. KRIEPS, Rosch, Humanheit.Ein Wegweiser zu Edward Steichens zeitloser Fotosammlung The Family of Man und deren Botschaft für das dritte Jahrtausend. Numéro spécial du Courrier de l'Education Nationale, Luxembourg, 2005, p. 11.

17 TOURNIER, Michel, Dieu est-il photographe?, in The Family of Man. Témoignages et documents, cit., p. 111.

${ }_{18}$ Jusqu'aujourd'hui les incertitudes sur la chronologie ne permettent pas de retracer le parcours exact de l'exposition. De même face aux différents chiffres avancés de visiteurs, il est difficile de réaliser une étude quantitative. 
de toute la tournée internationale ${ }^{19}$ et pas uniquement parce qu'un pavillon lui fut entièrement dédié.

Une troisième critique paraît à l'étude des images qui composent l'exposition : Edward Steichen ne crée pas de nouvelles photographies mais recourt à des images passées pour raconter une histoire, elles ne sont alors plus qu'illustratives. A cette décontextualisation des images, s'ajoute le travail de recadrage et la scénographie établissant de nouveaux rapports physiques entre les clichés, les plus grands retenant l'attention de la majeure partie des visiteurs. Le décalage entre le spectateur initié des années '50 et le "simple" visiteur d'alors était sans doute plus important que de nos jours $^{20}$. Bien que le lieu de la prise de vue, l'auteur de l'image et sa provenance aient toujours été mentionnés, rien ne transparait de l'histoire propre des images. Ainsi l'exposition a pu être plus facilement perçue dans son intégralité qu'aujourd'hui où une information plus importante autour de chaque cliché circule. Beaucoup de ceux retenus par Steichen ont eu un avant et un après indépendant de leur présence dans l'exposition. D'autres ont été en quelque sorte révélés par elle avant d'être réutilisés dans un contexte différent. Il est fait ici référence en particulier aux quatre images tirées de l'exposition et reprises par Pierre Bourdieu dans son étude Un art moyen, essai sur les usages sociaux de la photographie ${ }^{21}$. L'image déjà mentionnée plus avant de l'enfant juif du ghetto de Varsovie apparait quant à elle régulièrement dans les manuels d'histoire ${ }^{22}$.

Un autre reproche à l'encontre de l'exposition est d'avoir eu recours à des photographies de presse, le plus souvent des illustrés américains. Là se conjuguent deux critiques déjà citées, celle du réemploi de clichés et celles de propagation d'un modèle essentiellement occidental pour ne pas dire exclusivement américain. En se référant plus ou moins implicitement à Luc Boltanski dans l'ouvrage dirigé par Pierre Bourdieu, c'est la question de la symbolique de l'image photographique qui est posée ${ }^{23}$. Si l'image d'actualité est réellement prise sur le vif, comment peut-elle contenir les

19 SANDEEN, Eric J., Picturing an Exhibition. The Family of Man and 1950s America, Albuquerque, University of New Mexico Press, 1995, p. 125.

${ }^{20}$ Il ne s'agit pas ici de considérer les visiteurs des expositions ou les lecteurs de revues ou de magazines illustrés comme naïfs mais plutôt innocents. L'éducation aux médias n'était pas aussi développée qu'aujourd'hui. Mais déjà en décembre 1956 comme le rapporte Gisèle Freund l'hebdomadaire français L'Express avait publié une série de photographies en deux versions distinctes. Une fois l'ordre des images et les commentaires transformés, les propos se contredisaient. Cfr. FREUND, Gisèle, Photographie et société, Paris, Le Seuil, 1974, p. 155.

${ }^{21}$ BOURDIEU, Pierre (dir.), Un art moyen. Essai sur les usages sociaux de la photographie, Paris, Les Éditions de Minuit, 1965, illustrations 7 à 10.

${ }_{22}$ Cfr. ROUSSEAU, Frédéric, L'enfant juif de Varsovie: Histoire d'une photographie, Paris, Seuil, 2009.

${ }_{23}$ Cfr. BOLTANSKI, Luc, «La rhétorique de la figure», in BOURDIEU, Pierre (dir.), op. cit., pp. 173-188. 
symboles utiles à sa compréhension ou en adéquation avec le message à diffuser ? Celui véhiculé par la photographie composée repose par définition sur l'agencement des symboles. S'ensuit la problématique du cadre référentiel dans la mesure où, "The Family of Man » se veut une expression universelle de la confiance en l'Homme. Dans un espace mondialisé où les référents ont depuis longtemps dépassé les frontières, que les politiques ont eux-mêmes fait disparaitre, les différences d'appréciation se sont atténuées. Mais dans le milieu des années cinquante, dix ans à peine après la fin d'un conflit mondial pendant lequel chacun a travaillé à renforcer les symboliques nationales, il existait un nombre plus important de lectures de l'exposition que maintenant. Ainsi en témoigne la déclinaison de l'exposition primaire en plusieurs versions itinérantes - le nombre varie de cinq à neuf selon les auteurs ou les documents - même si à en croire l'introduction au numéro de février 1956 du Courrier de l'Unesco déjà cité plus avant «il s'en dégage une philosophie et un enseignement d'autant plus puissants que ces images parlent dans un langage compris de tous $»^{24}$.

\section{La patrimonialisation}

L'inscription de l'exposition le $1^{\text {er }}$ septembre 2003 au Registre de la Mémoire du Monde consacre en quelque sorte son caractère universel, tant mis en avant par ses promoteurs. Dans sa présentation l'Unesco assimile l'exposition à un fait historique, à un monument de la Guerre Froide:

The Family of Man, tout en étant devenu un monument légendaire de l'histoire de la photographie, dépasse de très loin le cadre d'une exposition dans le sens classique du terme. Elle peut être considérée comme la mémoire de toute une époque, marquée par la guerre froide et le Maccarthysme, mais aussi par les attentes et les désirs de paix de millions d'hommes et de femmes dans le monde entier25.

\footnotetext{
${ }^{24}$ Le Courrier de l'Unesco, cit., p. 3.

${ }^{25}$ Le texte de la page web du "Registre de la Mémoire du Monde" (voir note $\mathrm{n}^{\circ}{ }^{1}$ ) reprend intégralement la formulation de Jean Back dans le formulaire de proposition d'inscription accessible en ligne depuis la même page. URL :

<http://www.unesco.org/new/fileadmin/MULTIMEDIA/HQ/CI/CI/pdf/mow/nomination_for ms/Family\%20of\%20Man\%20\%20Nomination\%20FormFR.pdf > [consulté le 11 mars 2014].
} 


\subsection{L'exposition comme objet vs les objets de l'exposition}

«C'est dans les documents que reposent les témoignages les plus parlants des entreprises intellectuelles et culturelles de l'humanité» rappelait Dietrich Schüller dans un guide sur la conservation des documents édité en 1998 par l'UNESCO ${ }^{26}$. Le "document" dont il est question ici est une collection de photographies générée par Edward Steichen et son équipe. " The Family of Man » n'est pas, à la différence d'autres exemples comme «La collection de l'Empereur» ${ }^{27}$ inscrite simultanément au même registre qu'elle, un rassemblement de tirages originaux et rares mais le résultat d'un travail de recherche de matériau d'illustration : «Wayne Miller entered the Life archives in late 1953 to discover whether or not photographs could be found that captured the concepts that Steichen had dreamed of» 28 .

C'est le curateur qui a présidé au choix des clichés, à la détermination de leur format d'agrandissement et pour certains à leur recadrage. A l'exception des photographes Ansel Adams et Robert Frank ${ }^{29}$, aucune objection ne semble avoir été émise par les contributeurs retenus ou sollicités par Edward Steichen face à ce réemploi de leurs images. La présence en nombre de photographies issues de la presse illustrée occidentale dans l'exposition est peut-être à l'origine du son succès, souvent mis en avant et aussi retenu comme un critère important pour l'inscription au registre de la Mémoire du Monde. Il y a adéquation entre les espaces de provenances des images et ceux de leur présentation. A contrario « The Family of Man » n'a pas ou peu voyagé dans les espaces dont elle n'avait pas obtenu d'images, à l'exemple du continent africain très peu visité. Aujourd'hui à Clervaux, elle ne devrait pas attirer plus de visiteurs de ce continent qu'hier. Ce patrimoine est-il donc réellement universel?

${ }^{26}$ SCHÜLLER, Dietrich, Préface, in BOSTON, Georges (dir.), MEMOIRE DU MONDE: Guide des normes, pratiques recommandées et ouvrages de référence concernant la conservation des documents de toute nature. CII.98/WS/4, 1998, p. 3. URL:

< http://unesdoc.unesco.org/images/o011/o01126/112676fo.pdf > [consulté le 11 mars 2014]. 27 URL: < http://www.unesco.org/new/fr/communication-and-information/flagship-projectactivities/memory-of-the-world/register/full-list-of-registered-heritage/registered-heritagepage-8/the-emperors-collection-foreign-and-brazilian-photography-in-the-xixcentury/\#c186914 > [consulté le 11 mars 2014].

28 [Wayne Miller est entré dans les archives de Life en fin d'année 1953 pour y découvrir si, oui ou non, des photographies qui reflétaient les concepts que Steichen avait inventés, pouvaient y être trouvées]. SANDEEN, Eric, op.cit., p. 41.

${ }^{29}$ Cfr. SANDEEN, Eric, op.cit., p. 59 et le formulaire de proposition d'inscription (voir note $\mathrm{n}^{\circ} 3$ ), p. 6 . 


\subsection{Patrimoine étatsunien, luxembourgeois ou mondial ?}

«When Ann Fortescue was just six years old and growing up in New York City, she often paged through a coffee table art book entitled "The Family of Man"» a écrit Meredith Moss le 16 mars 2014 ${ }^{30}$. La journaliste nomme simplement l'exposition, ce qui conduit à penser que cette dernière reste une référence pour un public initié, même si l'image du catalogue bien en vue sur la table du salon semble quelque peu décalée par rapport au message de paix et de confiance en l'âme humaine que les créateurs puis ensuite les défenseurs de l'exposition lui reconnaissent.

Fred Turner prétend qu'il est facile de critiquer l'exposition, de la réduire à une propagande étatsunienne mais que cela ne correspond pas à la réalité du travail de Steichen ${ }^{31}$. Il présente même « The Family of Man » comme une création dans laquelle son auteur a cherché à replacer l'être humain en face de lui-même, lui démontrant d'une part l'universalité tout en conservant d'autre part les particularismes, donc les différences. Selon lui la scénographie est une preuve d'un anti-autoritarisme de Steichen, le curateur laissant au visiteur l'entière maittrise de son parcours à travers l'espace d'exposition ${ }^{32}$.

Plus que sur son cheminement, c'est surtout sur son rythme que le visiteur garde la mainmise33. Basée sur les travaux d'Herbert Bayer sur l'expérience visuelle, la mise en espace englobe le visiteur dans un monde d'images, le forçant à établir des relations entre-elles. Chaque individu crée alors un réseau qui lui est propre. Turner y voit là un argument à opposer à ceux qui pensent que l'exposition est un instrument de propagande. Même si la liberté évoquée par Turner peut rejoindre la conception de Roland Barthes, fervent critique de l'exposition, qui considère la lecture d'images publiques comme une affaire privée ${ }^{34}$, elle reste subordonnée à des schèmes de pensée

30 [Alors qu'Ann Fortescue avait tout juste six ans et grandissait à New-York, elle feuilletait souvent un livre d'art de table basse intitulé The Family of Man ]. MOSS, Meredith, "Famed photos by Ansel Adams now in Springfield », in Dayton Daily News, 16 mars 2014, Page E-1. URL : < http://daytondailynews.mycapture.com/mycapture/enlarge.asp?image $=50999218$ > [consulté le 18 mars 2014].

${ }^{31}$ TURNER, Fred, «The Family of Man and the Politics of Attention in Cold War America», in Public Culture, XXIV, 66, 1/2012, pp. 55-84.

32 « This heterogeneous form of installation asked viewers to follow their own course among the images [...] ». [Cette forme hétérogène d'installation a demandé aux spectateurs de suivre leur propre cheminement parmi les images]. TURNER, Fred, op. cit., p. 57.

33 Marc-Emmanuel Melon compare le parcours à suivre à « [...] un contre-film dans lequel, au contraire du dispositif classique de la salle obscure, le spectateur serait mobile et les images fixes ». MELON, Marc-Emmanuel, "The Family of Man ou le contre-film», in The Family of Man. Témoinages et documents, cit., pp. 55-56. Voir aussi DUPUIS, Claude, op. cit., p. 277.

34 Cfr. BARTHES, Roland, La chambre claire. Notes sur la photographie, Paris, Gallimard, 1980, pp. 151-154. 
acquis par les visiteurs. Quand aujourd'hui l'éducation aux média relève de plus en plus de l'éducation formelle, les visiteurs des années '50 et '60 étaient probablement dans une approche plus informelle et plus influencés par cette presse s'adressant aux classes moyennes à gros renfort d'images. Dans la scénographie de l'exposition, qu'elle fût influencée par les théories de Beyer ou les précédentes réalisations de Steichen à l'exemple de l'exposition Road to Victory durant la seconde guerre mondiale, le visiteur retrouve à la fois les lay-out mais aussi beaucoup de clichés de cette même presse illustrée. En adoptant une version brochée du catalogue (a coffee table art book35) les créateurs ont délibérément ciblé la "middle class" américaine tout d'abord, puis internationale par la suite.

Poser la question de la "nationalité" d'un tel patrimoine peut paraitre de prime abord inapproprié. Cette approche a néanmoins permis dans un premier temps de distinguer les usages par les différentes collectivités de cette collection photographique avant d'envisager maintenant les divers enjeux de sa patrimonialisation, notamment celle dans le cadre de l'UNESCO.

Depuis 1964 la création d'Edward Steichen et son équipe au sein du Museum of Modern Art de New-York est la propriété du Grand-Duché du Luxembourg. Comme pour les autres documents inscrits au Registre de la «Mémoire du Monde» l'exposition «The Family of Man » appartient juridiquement au pays et/ou à l'institution dépositaire qui a formulé la demande d'inscription. En 1994 Rosch Krieps est revenu sur les relations difficiles entre Edward Steichen et le Grand-Duché : l'enfant du pays né en 1879 et émigré avec ses parents aux Etats-Unis en 1891 n'a pas entretenu de relation avec son pays de naissance avant les années '50. C'est seulement en 1953 à l'âge de 74 ans et dans le cadre de la préparation de l'exposition qu'il revint au Luxembourg et présenta son projet aux autorités qui l'ignorèrent poliment 36 .

Les raisons et les initiateurs du don ne semblent pas clairement identifiés. Comme il a été déjà signalé au départ de cette contribution, c'est Edward Steichen qui aurait proposé le Château de Clervaux, au nord du Grand-Duché, comme lieu d'accrochage de la dernière version itinérante de l'exposition. Après une présentation au public en 1966, la collection photographique y est restée en partie accrochée jusqu'en 1975 puis a été stockée jusqu'au début des années 1990 avant d'être restaurée puis exposée à nouveau et de manière permanente à Clervaux à partir de juin 1994. Ni argument ni document ne viennent appuyer l'idée d'une appropriation à caractère «nationaliste » de

\footnotetext{
35 Cfr. note 30.

${ }^{36}$ Cfr. KRIEPS, Rosch, «Luxembourg 1966. Un émigré en quête de patrie», in The Family of Man. Témoinages et documents, cit., pp. 163-174.
} 
l'exposition par le Grand-Duché de Luxembourg. Le message caractérisé comme humaniste correspond néanmoins aux préoccupations humanitaires de cet état 37 , signataire du Traité de Bruxelles en 1948, membre fondateur de l'OTAN en 1949 et à l’origine de la communauté économique européenne en 1957.

Un des enjeux avoué pour le gouvernement du Grand-Duché fut de «concrétiser d'abord sa politique de décentralisation en faveur des régions qui sont restées à l'écart de la vie culturelle du pays [...]»38. L'inscription des vieux quartiers et fortifications de la Ville de Luxembourg en 1994 sur la liste du Patrimoine Mondial de l'UNESCO, l'ouverture au public de «The Family of Man » en juin 1994, quelque mois avant le démarrage de «Luxembourg '95»39, démontrent comment dans les années 1990 le Luxembourg a mis en place une politique culturelle qui lui a permis de valoriser et faire connaitre ses collections mais aussi de se doter d'une image plus gratifiante que celle peu flatteuse - de paradis fiscal. La visibilité du patrimoine luxembourgeois a été renforcée au plan européen et mondial par la labellisation UNESCO. Pour la deuxième fois Capitale Européenne de la Culture en 2007, Luxembourg et le Grand-Duché ont poursuivi depuis le début des années 2000 une politique culturelle plus dévolue à la promotion d'une identité luxembourgeoise que dans les années précédentes, tout en s’inscrivant dans le contexte plus vaste de la Grande Région ${ }^{40}$.

«L'exposition "The Family of Man" peut, [...], nous aider à comprendre ce qu'étaient la famille et la société américaines des années cinquante»41. Dans une approche socio - ou ethnologique de l'exposition, il faut distinguer deux catégories principales d'artéfacts présentés au public: la première est celle des photographies, la seconde est l'exposition et son catalogue. Ils sont la synthèse du choix des images et de

37 « Le Luxembourg témoigne de son engagement pour l'ONU, ses buts et ses principes en remplissant non seulement ses obligations financières en vertu de la charte, mais en contribuant également au financement volontaire des fonds et programmes des Nations unies, en participant aux opérations de maintien de la paix et en poursuivant une politique active de coopération au développement». URL:

< http://www.gouvernement.lu/1719301/ue-organisations-internationales > [consulté le 27 mars 2014].

$3^{8}$ SANTER, Jacques, Préface. Un appel au respect de la dignité humaine, in The Family of Man. Témoinages et documents, cit., p. 5.

39 Luxembourg fut en 1995 Ville Européenne de la Culture. À cette occasion le «Casino Luxembourg » accueilli du 15 juillet au 27 août 1995 deux expositions photographiques « Face to Face » rassemblant des œuvres personnelles de Steichen et « The Bitter Years » une exposition réalisée par Steichen en 1962 à partir de clichés de la FSA, Farm Security Administration, et offerte par le MoMA au Grand-Duché de Luxembourg en 1967.

${ }^{40}$ Cfr. le site Arts et Culture au Luxembourg crée en 2009 qui rassemble, en plus des nombreux liens, beaucoup d'informations sur l'offre culturelle du pays. URL :

< http://culture.luxembourg.public.lu/SIP-Culture.42-2.html > [consulté le 26 mars 2014].

${ }^{41}$ SÉGALEN, Martine, The Family of Man ou la grande illusion. Quel regard peut bien porter l'ethnologue sur une telle exposition?, in The Family of Man. Témoinages et documents, cit., p. 119. 
la scénographie. Les images retenues par Steichen ont été choisies pour leur intérêt visuel en même temps que dissociées de leur contexte originel, ce qui leur confère un nouvel état "d'objet" dans l'exposition. Seule une petite partie des images illustrent les thèmes de la famille et de la société étatsuniennes des années '50. Le visiteur doit donc procéder par comparaison ou association afin de déterminer les spécificités énoncées par l'ethnologue Martine Ségalen. C'est ici qu'intervient la liberté supposée du public exprimée par Fred Turner mais surtout que se confrontent les référentiels du curateur et du visiteur. La forme de présentation choisie par Edward Steichen relève de ce que Barbara Kirshenblatt-Gimblett définit comme "In Context », un mode d'exposition dans lequel le curateur impose un cadre assez stricte au public ${ }^{42}$. Une des difficultés imposées aux visiteurs par Steichen et son équipe est d'être confronté à l'humain à travers des photographies. Certains aspects de la scénographie font ouvertement référence aux expositions universelles ou coloniales au cours desquelles des êtres humains issus de cultures et de contrées différentes étaient exposés au regard du visiteur. Aujourd'hui largement dénoncé mais pas complètement éradiqué, le recours à l'exotisme peut être interprété comme la formulation d'un sentiment de supériorité. Nombreux sont ceux qui ont reconnu en « The Family of Man » une forme d'expression de l'hégémonie américaine au sortir de la seconde guerre mondiale et durant la Guerre Froide.

L'avant-dernière image que le visiteur perçoit avant d'être plongé dans une ultime section aux nombreuses photographies d'enfants plutôt heureux est celle d'une réunion de l'ONU, signée Maria Bordy. Accompagnée d'un extrait de la Charte des Nations Unies ce cliché renvoie aux espoirs fondés par Steichen en cette nouvelle organisation crée au sortir du second conflit mondial pour épargner de nouvelles guerres aux futures générations. Cette photographie fait face à celle d'une explosion atomique caractérisée par son «champignon », une image qui n'a jamais été reproduite dans le catalogue. L'UNESCO a, notamment dans son «Courrier» de février 1956, participé à la promotion de «The Family of Man »43. Quel était alors le lectorat de cette revue et existe-t-il une corrélation entre ces lecteurs et les visiteurs de l'exposition ? A l'heure actuelle face au manque d'enquêtes et de statistiques sur ce sujet, personne ne peut

42 « In-context approaches exert strong cognitive control over the objects, asserting the power of classification and arrangement to order large numbers of artifacts from diverse cultural and historical settings and to position them in relation to one another.» [Les approches "In-context" exercent un contrôle cognitif fort sur les objets, affirmant la puissance de classification et d'arrangement à ordonner un grand nombre d'artefacts provenant de différents contextes culturels et historiques et à les agencer les uns par rapport aux autres]. KIRSHENBLATTGIMBLETT, Barbara, Object of Ethnography, in ID., Destination Culture. Tourism, Museums, and Heritage, Berkeley, University of California Press, 1998, pp. 21-22.

43 Cfr. note 14. 
prétendre répondre à ces interrogations. Reste l'humanisme. Cette valeur aux contours variables est au centre des préoccupations de l'organisation, dont l'un des slogans affichés est «construire la paix dans l'esprit des hommes et des femmes ». Dans ce cadre et face aux explications avancées sur le projet initial d'Edward Steichen, en particulier celle de témoigner de la capacité humaine à dépasser les conflits et proposer un monde plus ouvert à ses descendants, l'exposition peut être considérée comme l'un des meilleurs outils pédagogiques de l'UNESCO pour promouvoir son action à travers le monde. Son inscription au "Registre de la Mémoire du Monde" n'en est que la suite logique. Ce patrimoine parait alors bien moins américain qu'onusien bien qu'il existe de nombreuses interdépendances entre l'exposition, le MoMA, l'organisation internationale et la fondation Rockefeller.

\section{Conclusion}

S'il n'est pas politiquement correct de parler de propagande à propos de l'UNESCO, ce terme n'est peut être complètement écarté à l'égard de l'exposition « The Family of Man ». Les critiques adressées au projet de Steichen font écho aux définitions de Hobsbwam, Lotze ou Benjamin rappelées dans l'appel à contribution44 du numéro 18 de Diacronie consacré aux expositions universelles et à leurs rapports aux constructions identitaires45. Si dans le cas de l'assemblage photographique dont il est fait état ici il n'est pas clairement question d'afficher une nationalité particulière, le message supposé de son créateur repose sur des fondements religieux judéo-chrétiens et une vision quasi-exclusivement occidentale de l'humanité. Créée dans les années cinquante à New-York et n'ayant au départ pas vocation à parcourir le monde mais uniquement les Etats-Unis, elle aurait pu rester un phénomène américano-américain. Avec une scénographie adaptée d'expériences diverses (Beyer, Road to Victory...) mais aussi très proche des mises en page de la presse illustrée, l'exposition tend à se confondre avec cette même presse. En se confrontant à d'autres espaces, elle s'est retrouvée à la fois face à un large public conquis - au propre comme au figuré - mais aussi confrontées à quelques critiques persistantes. Elle représente encore aujourd'hui « une certaine conception américaniste du monde ${ }^{46}$. Si dans une perspective historique The Family

\footnotetext{
${ }^{44}$ Cfr. appel à contribution, URL : < http://www.studistorici.com/2013/11/o9/cfp18_it > [consulté le 31 mars 2014].

${ }^{45}$ Diacronie. Studi di Storia Contemporanea : Le esposizioni: propaganda e costruzione identitaria , 18, 2/2014, http://www.studistorici.com/2014/06/29/n-18-giugno-2014/ [consulté le 13 août 2014].

${ }^{46}$ MELON, Marc-Emmanuel, op. cit., p. 56.
} 
of Man ressemble en beaucoup de points à une exposition universelle «traditionnelle », elle s'en distingue néanmoins par le fait qu'elle existe encore dans son intégralité. Généralement la connaissance des expositions, pas uniquement universelles, se fait par l'exploitation de documents anciens (catalogue, presse, photographies ou encore monuments et pavillons nationaux) qui ont quelquefois permis une reconstitution même partielle comme ce fut le cas pour l'exposition « Entarte Kunst »47, dont de nombreuses œuvres ont été vendues, perdues ou détruites pendant la seconde guerre mondiale. La version de « The Family of Man » visible de manière durable à Clervaux n'est pas à proprement parlé une reconstitution même s'il y a eu rupture de la présentation dans le temps entre 1966 et 1994. Dans une conception linéaire et ininterrompue de la vie de l'exposition photographique depuis les années cinquante à travers sa présentation, son catalogue maintes fois réédité et son classement comme document de la Mémoire du Monde, c'est l'aspect revendicatif inculqué par son auteur et ses promoteurs qui est perpétué. L'inscription à l'UNESCO confère à l'exposition à la fois un caractère universel mais aussi exceptionnel. Dans un futur sans doute plus proche que prévu, force sera de constater que la surabondance de labellisation peut nuire à la promotion des objets qu'elle est censée protéger.

A l'écart de toute idéologie humaniste, religieuse, pro- ou anti-américaine, il est pourtant possible de considérer « The Family of Man » comme un artefact, par essence dissocié de son contexte originel, aujourd'hui exposé comme un simple document d'histoire aux qualités esthétiques satisfaisantes. Alors que les diverses polémiques relatées ici suggèrent que les deux approches seraient inconciliables, il faut espérer que de nouvelles études permettront d'aborder l'exposition sous d'autres angles. Yoko Tsuchiyama a entrepris l'étude du traitement de la technologie nucléaire dans les différentes versions de l'exposition ${ }^{48}$. Elle s'est concentrée sur les images qui présentent les dévastations et dégâts ainsi que sur la construction narrative dont elles font partie. Cette narration est cependant plus vaste que celle proposée jusqu'ici. Elle englobe des images d'autres sections que celles retenues par Tsuchiyama ${ }^{49}$. Une étude plus générale

47 L'exposition munichoise de 1937 a été reconstituée par le Los Angeles County Museum of Art et présentée en 1991 à Los Angeles, Chicago et Washington D. C. avant d'être montrée à Berlin au printemps 1992. BARRON, Stephanie (dir.), „Entarte Kunst“ Das Schicksal der Avant-Garde im Nazi-Deutschland, München,Hirmer, 1992.

48 TSUCHIYAMA, Yoko, La réinterprétation de "The Family of Man" (V. O. MoMA, 1955) lors de son remontage dans les années 90 - autour de l'exposition sur la bombe atomique. URL: $<$ http://halshs.archives-

ouvertes.fr/docs/oo/67/05/73/PDF/La_rA_interprA_tation_de_The_Family_of_Man_Yoko_ Tsuchiyama.pdf> [consulté le 31 mars 2014].

49 Les portraits d'Albert Einstein par Ernst Haas et de Robert Oppenheimer par Alfred Eisenstaedt ainsi que l'image de physiciens nucléaires prise à l'Université de Californie par Nat Farbman dans la section de l'exposition dédiée à l'éducation appartiennent tout autant à cette 
du traitement narratif et iconographique de conflits dans l'exposition - l'image la plus ancienne du corpus, datée autour de 1861, est une photographie de la guerre de sécession par Mathew Brady - reste donc d'actualité.

narration. Dans une autre section consacrée à la maison et au bureau une image très graphique de circulation de particules sert de fond à la présentation des photographies. Avec cet agencement le curateur parvient à démontrer l'importance de cette nouvelle technologie que représente le nucléaire, sans jamais avoir à la présenter directement. Dans le catalogue un extrait de texte de la commission de l'énergie atomique américaine accompagne le groupe d'images. 
«The Family of Man»: réflexions autour des usages et de la patrimonialisation d'une exposition photographique controversée

\section{* L'auteur}

Claude Dupuis est diplômé de l'Université de Trèves (Allemagne) et docteur en Histoire de l'Art. Après une thèse en 2007 sur la place de l'église Saint Jacques de Dieppe dans l'architecture religieuse en Normandie entre les 12e et 16e siècles (Monasterium Sancti Jacobi. Die Baugeschichte von Saint-Jacques zu Dieppe im Spannungsfeld der normannischen Architektur des 12. bis 16. Jahrhunderts, Dissertation, Universität Trier, Trier 2007, URL : $<$ http://ubt.opus.hbz-

nrw.de/volltexte/2013/785/pdf/dieppe_vollstAndiger_online_version.pdf $>$ ), ses recherches en dehors de tout rattachement institutionnel portent aujourd'hui encore sur l'architecture gothique, mais aussi sur l'iconographie des monuments aux morts français. Parallèlement, dans une approche transdisciplinaire (histoire de l'art, sociologie visuelle), il poursuit ses investigations sur la formation du regard, en particulier celui des photographes. C'est ainsi que dans un esprit analogue, il porte régulièrement son intérêt sur la reconnaissance et la reproduction de schémas constructifs en photographie à travers les époques.

URL: < http://www.studistorici.com/progett/autori/\#Dupuis >

\section{Per citare questo articolo:}

DUPUIS, Claude, «"The Family of Man": réflexions autour des usages et de la patrimonialisation d'une exposition photographique controversée», Diacronie. Studi di Storia Contemporanea, 29/09/2014, URL:<http://www.studistorici.com/2014/09/29/dupuis_numero_19/ >

Diacronie Studi di Storia Contemporanea $\widehat{\beta}$ www.diacronie.it

Risorsa digitale indipendente a carattere storiografico. Uscita trimestrale. redazione.diacronie@hotmail.it

Comitato di redazione: Jacopo Bassi - Luca Bufarale - Elisa Grandi - Deborah Paci - Fausto Pietrancosta - Matteo Tomasoni - Luca Zuccolo

Diritti: gli articoli di Diacronie. Studi di Storia Contemporanea sono pubblicati sotto licenza Creative Commons 2.5 Possono essere riprodotti a patto di non modificarne i contenuti e di non usarli per fini commerciali. La citazione di estratti è comunque sempre autorizzata, nei limiti previsti dalla legge. 